\title{
Dosimetric Parameters Predicting Tooth Loss after Carbon Ion Radiotherapy for Head and Neck Tumors
}

\author{
Nobuteru Kubo ${ }^{1, *} \mathbb{D}^{\mathbb{D}}$, Makoto Sakai $^{1}{ }^{1}$, Hidemasa Kawamura ${ }^{1} \mathbb{D}$, Takahiro Oike ${ }^{1}$, Yoshiki Kubota ${ }^{1}$, \\ Mai Anakura ${ }^{1}$, Akiko Adachi ${ }^{1}$, Hiro Sato ${ }^{1} \mathbb{D}$, Atsushi Musha ${ }^{1,2}{ }^{\mathbb{D}}$, Naoko Okano ${ }^{1}$, Takuya Kaminuma ${ }^{1}$, \\ Katsuyuki Shirai ${ }^{1,3}$, Jun-ichi Saitoh ${ }^{1,4}$, Satoshi Yokoo ${ }^{2}$, Kazuaki Chikamatsu ${ }^{(D)}$ and Tatsuya Ohno ${ }^{1}$ \\ 1 Gunma University Heavy Ion Medical Center, 3-39-15, Showa-machi, Maebashi 371-8511, Japan; \\ sakai-m@gunma-u.ac.jp (M.S.); kawa@gunma-u.ac.jp (H.K.); oiketakahiro@gunma-u.ac.jp (T.O.); \\ y_kubota@gunma-u.ac.jp (Y.K.); m1920019@gunma-u.ac.jp (M.A.); akiadachi@gunma-u.ac.jp (A.A.); \\ hiro.sato@gunma-u.ac.jp (H.S.); musha@gunma-u.ac.jp (A.M.); okano.n@gunma-u.ac.jp (N.O.); \\ cami_taku@gunma-u.ac.jp (T.K.); kshirai@jichi.ac.jp (K.S.); junsaito@med.u-toyama.ac.jp (J.-i.S.); \\ tohno@gunma-u.ac.jp (T.O.) \\ 2 Department of Oral and Maxillofacial Surgery, and Plastic Surgery, Gunma University Graduate School of \\ Medicine, 3-39-15, Showa-machi, Maebashi 371-8511, Japan; syokoo@gunma-u.ac.jp \\ 3 Department of Radiology, Jichi Medical University Hospital, 3311-1, Yakushiji, Shimotsuke 329-0498, Japan \\ 4 Division of Radiation Oncology, Department of Radiology, Faculty of Medicine, Academic Assembly, \\ University of Toyama, 2630, Sugitani, Toyama 930-0194, Japan \\ 5 Department of Otolaryngology-Head and Neck Surgery, Gunma University Graduate School of Medicine, \\ 3-39-15, Showa-Machi, Maebashi 371-8511, Japan; tikamatu@gunma-u.ac.jp \\ check for \\ * Correspondence: kubo@gunma-u.ac.jp; Tel.: +81-27-220-8383
} updates

Citation: Kubo, N.; Sakai, M.; Kawamura, H.; Oike, T.; Kubota, Y.; Anakura, M.; Adachi, A.; Sato, H.; Musha, A.; Okano, N.; et al. Dosimetric Parameters Predicting Tooth Loss after Carbon Ion Radiotherapy for Head and Neck Tumors. Radiation 2021, 1, 183-193. https://doi.org/10.3390/ radiation1030017

Academic Editor: David A. Gewirtz

Received: 23 March 2021

Accepted: 6 July 2021

Published: 9 July 2021

Publisher's Note: MDPI stays neutral with regard to jurisdictional claims in published maps and institutional affiliations.

Copyright: (c) 2021 by the authors. Licensee MDPI, Basel, Switzerland. This article is an open access article distributed under the terms and conditions of the Creative Commons Attribution (CC BY) license (https:// creativecommons.org/licenses/by/ $4.0 /)$.
Simple Summary: Head and neck non-squamous cell carcinomas are predominantly located in the sinonasal cavities. Loss of the teeth located adjacent to the sinonasal cavities is one adverse event experienced due to post-carbon ion radiotherapy (CIRT) for head and neck tumors. Tooth loss reduces quality of life (QOL) by causing masticatory dysfunction, swallowing dysfunction, and changes in appearance. The aim of our study was to elucidate the incidence of tooth loss post-CIRT and to identify risk-predictive dosimetric parameters. The median time of tooth loss was 38.6 months. The volume of irradiated teeth was significantly greater for lost teeth than for remaining teeth throughout the dose range. The risk-predictive parameters found in this study could be used (i) to spare teeth and thereby maintain patient QOL or (ii) to predict which tooth will be lost after CIRT and extract them before CIRT since this can prevent the development of sequestrum-associated chronic pain.

Abstract: Background: Tooth loss reduces quality of life; however, little is known about tooth loss caused by carbon ion radiotherapy (CIRT). Here, we aimed to elucidate the incidence of tooth loss post-CIRT for head and neck tumors and to identify risk-predictive dosimetric parameters. Methods: This study enrolled 14 patients (i.e., 171 teeth in total) with head and neck non-squamous cell carcinoma. All patients received CIRT comprised of 57.6 or $64.0 \mathrm{~Gy}$ (relative biological effectiveness, $\mathrm{RBE}$ ) in 16 fractions. Dose-volume analysis of the teeth was performed using receiver operating characteristic (ROC) curve analysis with VX (i.e., the volume irradiated with X Gy (RBE)). Results: The median follow-up period was 69.1 months. The median time of tooth loss was 38.6 months. The 5 year cumulative incidence of tooth loss was $13.3 \%$. The volume of irradiated teeth was significantly greater for the lost teeth than for the remaining teeth throughout the dose range. Using the cut-offs calculated from ROC curve analysis, V30-V60 showed high accuracy (i.e., >94\%) for predicting tooth loss, with V50 being the most accurate (cut-off, 58.1\%; accuracy, 0.95). Conclusions: This is the first report to examine the incidence of tooth loss post-CIRT and to identify risk-predictive dosimetric parameters.

Keywords: tooth loss; carbon ion radiotherapy; head and neck tumors; non-squamous cell carcinoma; dose-volume histogram 


\section{Introduction}

Radiotherapy, which is a standard treatment for cancer, is often performed using photons. Although photon radiotherapy has developed significantly over the past decade, some tumors are photon-resistant and some patients suffer severe adverse events [1,2]. Carbon ion radiotherapy (CIRT) has several advantages over photon radiotherapy, including a greater cell killing effect and superior dose distribution [3,4]. Taking advantage of these features, CIRT is used to treat various cancers such as lung cancer, head and neck cancer, sarcoma, prostate cancer, and pancreatic cancer. The therapeutic outcome of CIRT for these cancers is favorable and safe [5-9]. In particular, CIRT is used to treat tumors arising in the head and neck regions, mainly non-squamous cell carcinoma (NSCC) [10-13]. Most head and neck tumors treated with photon radiotherapy consist of squamous cell carcinoma (SCC) $[14,15]$. One of the standard therapies of SCC is photon radiotherapy with or without chemotherapy. NSCC is a relatively rare cancer in this region. There is no established standard of care for NSCC, which has characteristics different from those of NSCC. For example, NSCC is located predominantly in the sinonasal cavities [16]. By contrast, head and neck SCCs are located predominantly in the oral cavity, larynx, and pharynx [6]. This difference in primary tumor site according to histological subtype contributes to differences in adverse events (AEs) caused by CIRT and photon radiotherapy. For example, previous studies report maxillary osteonecrosis, vision loss, brain necrosis, and nasolacrimal duct obstruction after CIRT for head and neck NSCCs associated with the sinonasal cavities [17-20]; these AEs are rare after the application of photon radiotherapy.

Loss of the teeth located adjacent to the sinonasal cavities is one post-CIRT AE experienced by patients with head and neck tumors. Tooth loss reduces quality of life (QOL) by causing masticatory dysfunction, swallowing dysfunction, and changes in appearance. However, the incidence of tooth loss post-CIRT for head and neck tumors, as well as the dosimetric parameters that predict this AE remain unidentified. In order to address this issue, we performed a prospective dose-volume analysis of individual teeth in patients with head and neck NSCC treated with CIRT.

\section{Materials and Methods}

\subsection{Patients}

The present study is a predesigned subgroup analysis of a prospective cohort of patients undergoing CIRT for head and neck NSCC. The design and inclusion criteria for the original study were reported previously [16]. The inclusion criteria specific for the present study were as follows: (i) at least one tooth received CIRT; and (ii) the patient was followed-up by computed tomography (CT), magnetic resonance imaging (MRI), or panoramic radiography for at least 3 years. Of the 35 patients registered in this prospective study, 21 were excluded from this analysis (11 patients did not receive irradiation for a tooth and 10 had an insufficient follow-up period); therefore, 14 were included in total. This study was approved by the Institutional Review Board of Gunma University Hospital as an Opt-Out consent model (Number: HS2019-191).

\section{2. $C I R T$}

CIRT was performed as described previously. Briefly, patients were positioned in customized cradles (Moldcare; Alcare, Tokyo, Japan) and immobilized using thermoplastic shells (Shellfitter; Kuraray, Osaka, Japan). A mouthpiece was created to maintain the lower jaw in the correct position. CT images ( $2 \mathrm{~mm}$ slice thickness) were acquired and used to plan treatment. The voxel dimensions of all CT images were approximately $0.88 \times 0.88 \times$ $2.0 \mathrm{~mm}$. Contrast-enhanced MRI was performed and then the images were used to assist target delineation. Two clinical target volumes (CTV1 and CTV2) were delineated; CTV1 encompassed the whole anatomical site of the tumor origin (e.g., nasal cavity or maxillary sinus) whereas CTV2 encompassed the tumor. Two planning target volumes (PTV1 and PTV2) were created, with $2 \mathrm{~mm}$ margins around CTV1 and CTV2, respectively. 
The carbon ion dose was prescribed in terms of the unit Gy (relative biological effectiveness, RBE), which was calculated by multiplying the physical dose (Gy) by the RBE value for carbon ions (RBE values for carbon ions were obtained using the biological NIRS-model, which is based on a linear-quadratic model) [21]. In principle, each patient received 64.0 Gy (RBE) in 16 fractions; $57.6 \mathrm{~Gy}$ (RBE) in 16 fractions was used in cases where the tumor was adjacent to the skin or mucosa. In all cases, PTV1 received 36 Gy (RBE) and PTV2 received the remaining dose.

\subsection{Assessment of Tooth Loss}

Before CIRT, a dentist performed a base-line examination and the number of teeth was recorded. Patients received post-CIRT follow-up every month for the first 6 months and then every 3 months thereafter. MRI and CT scans were obtained alternately every 3 months and 18-fluorodeoxyglucose positron emission tomography was performed every year. Panoramic radiographs were obtained from dentists. The number of teeth was obtained primarily from CT (supported by MRI and panoramic radiographs). The followup period was calculated from the first day of irradiation to the day of imaging that revealed tooth loss.

\subsection{Dose-Volume Analysis of the Teeth}

Teeth in the treatment planning CT images were delineated and analyzed as individual objects. In this case, the definition of a tooth included the root part but not the crown part. Maxillary teeth but not mandibular teeth were analyzed. Dose-volume analysis was performed using MIM Maestro (version 6.8.7.; MIM Software, Inc., Cleveland, $\mathrm{OH}$, USA), with the dose-volume parameter VX, i.e., the volume of the teeth irradiated with $\mathrm{X}$ Gy (RBE).

\subsection{Statistical Analysis}

The cumulative incidence of tooth loss was evaluated using the Kaplan-Meier method and differences were assessed using a log-rank test. Cut-off values for VX to predict post-CIRT tooth loss were determined using receiver operating characteristic (ROC) curve analysis. The Smirnov-Grubbs' test was used to evaluate outlier values. Differences in numerical variables were examined using the Mann-Whitney test. A $p$-value $<0.05$ was considered statistically significant. All statistical analyses were performed using SPSS (version 25; SPSS Inc., Chicago, IL, USA).

\section{Results}

\subsection{Patient Characteristics}

The present study enrolled 14 patients (Table 1) and 171 teeth identified on preCIRT examination were analyzed. Primary tumor sites were located mainly in the nasal cavity and maxillary sinus, which are adjacent to the maxillary teeth. Adenoid cystic carcinoma was the most common histological type. Most patients received a dose of 64.0 Gy (RBE), delivered in 16 fractions. The median follow-up period was 69.1 months (range, 38.6-96.5 months). During follow-up, loss of 25 teeth was observed. One of the 25 lost teeth was excluded for the analysis because the $p$-value assessed by the SmirnovGrubbs' test was below $<0.05$ for V10, V20, V30, V40, V50, and V60, suggesting that the loss of this tooth was unrelated to CIRT (Supplementary Figure S1). Figure 1 shows a representative case of tooth loss; at 5 years post-CIRT, a part of the alveolar bone of the maxilla, which received high-dose carbon ions, disappeared and resulted in the loss of four teeth. 
Table 1. Patient characteristics.

\begin{tabular}{|c|c|c|}
\hline & Characteristics & All $(n=14)$ \\
\hline \multicolumn{3}{|l|}{ Age } \\
\hline & Median (range) & $59(32-77)$ \\
\hline \multicolumn{3}{|c|}{ Gender } \\
\hline & Male & 5 \\
\hline & Female & 9 \\
\hline \multicolumn{3}{|c|}{ Performance status } \\
\hline & 0 & 6 \\
\hline & 1 & 8 \\
\hline \multicolumn{3}{|c|}{ Histology } \\
\hline & Adenoid cystic carcinoma & 10 \\
\hline & Mucoepidermoid carcinoma & 2 \\
\hline & Olfactory neuroblastoma & 1 \\
\hline & Basal cell adenocarcinoma & 1 \\
\hline \multicolumn{3}{|c|}{ Primary tumor site } \\
\hline & Nasal cavity & 7 \\
\hline & Maxillary sinus & 3 \\
\hline & Oral cavity & 2 \\
\hline & Pharynx & 1 \\
\hline & Parotid gland & 1 \\
\hline \multicolumn{3}{|c|}{ Operability } \\
\hline & Operable & 8 \\
\hline & Inoperable & 6 \\
\hline \multicolumn{3}{|c|}{ Primary or recurrence } \\
\hline & Primary tumor & 12 \\
\hline & Postoperative recurrence & 2 \\
\hline \multicolumn{3}{|c|}{ T stage } \\
\hline & $\mathrm{T} 2$ & 2 \\
\hline & $\mathrm{T} 3$ & 6 \\
\hline & $\mathrm{T} 4$ & 6 \\
\hline \multicolumn{3}{|c|}{$\mathrm{N}$ stage } \\
\hline & No & 14 \\
\hline \multicolumn{3}{|c|}{ Carbon-ion dose } \\
\hline & 64.0 Gy (RBE)/16 fractions & 13 \\
\hline & 57.6 Gy (RBE)/16 fractions & 1 \\
\hline
\end{tabular}

RBE, relative biological effectiveness.

(A)
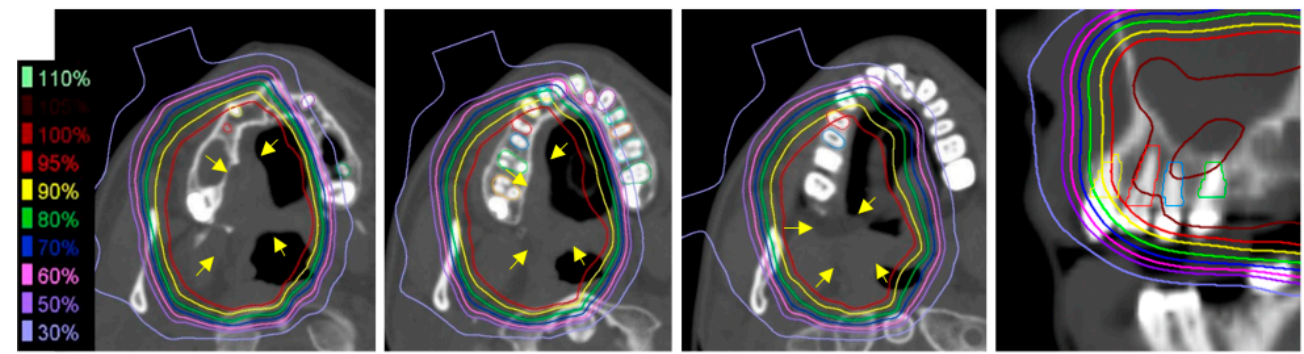

Figure 1. Cont. 
(B)
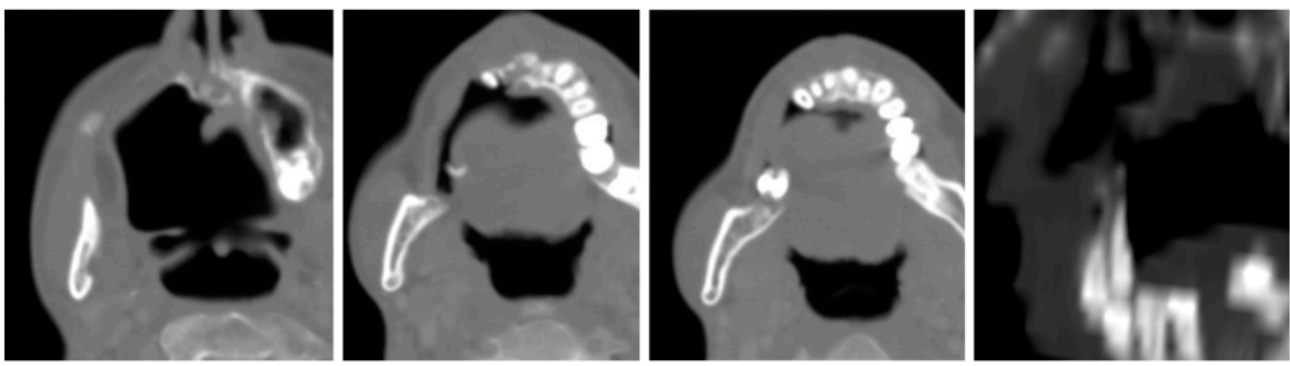

Figure 1. A representative patient with mucoepimoid carcinoma (yellow arrow) of the hard palate who experienced tooth loss after carbon ion radiotherapy (CIRT) with 64 Gy (relative biological effectiveness) delivered in 16 fractions. (A) Treatment planning computed tomography (CT) images showing isodose lines and the contours of the teeth (the three left-most images are axial sections and the right-most image is a coronal secton). (B) CT images taken 5 years post-CIRT.

\subsection{Incidence of Tooth Loss Post-CIRT}

Kaplan-Meier analysis revealed that the cumulative incidence of tooth loss showed a linear increase up to 5 years post-CIRT and then plateaued thereafter (Figure 2). The median time for occurrence of tooth loss post-CIRT was 38.6 months (range, 12.4-63.0 months). The 5 year cumulative incidence of tooth loss was $13.3 \%$. The number of tooth lost per period was $0(0 \%)$ at $0-12$ months, five (21\%) at $12-24$ months, four (17\%) at 24-36 months, seven $(29 \%)$ at $36-48$ months, six (25\%) at 48-60 months, two (8\%) at 60-72 months, and $0(0 \%)$ at 72 months. In this study, most tooth loss occurred within the period of 12 to 60 months post-CIRT.

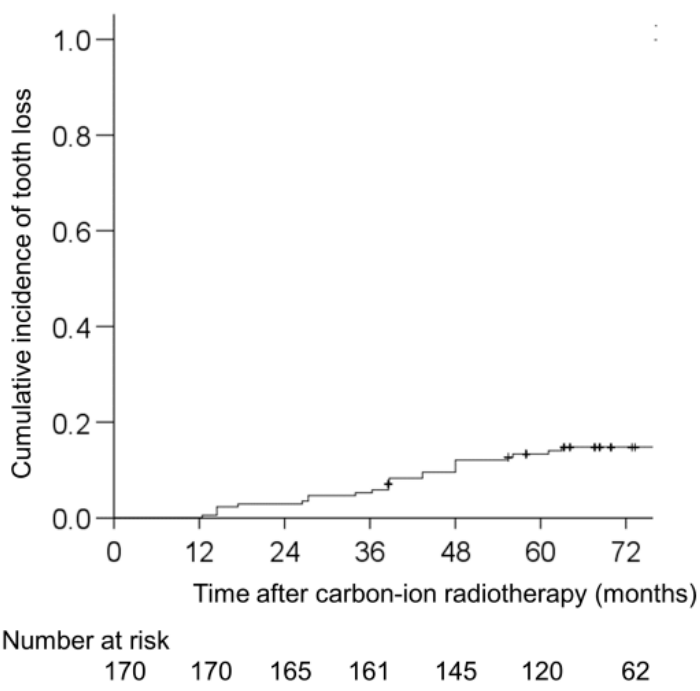

Figure 2. Cumulative incidence of tooth loss after carbon ion radiotherapy in patients with head and neck non-squamous cell carcinoma.

\subsection{Dose-Volume Analysis of Tooth Loss Post-CIRT}

The median volume of the 171 analyzed teeth was $0.28 \mathrm{~mL}$ (range, $0.08-1.00 \mathrm{~mL}$ ). The volumes of the lost teeth irradiated with carbon ions were significantly greater than those of the remaining teeth throughout the dose range ( $p<0.001$ for V10, V20, V30, V40, V50, and V60) (Figure 3). The maximum doses delivered to the lost teeth were significantly greater than those delivered to the remaining teeth (64.7 $\pm 0.5 \mathrm{~Gy}$ (RBE) vs. $24.1 \pm 20.7 \mathrm{~Gy}$ (RBE), $p<0.001)$. The mean dose delivered to the lost teeth was also significantly greater 
than that delivered to the remaining teeth $(62.3 \pm 3.6 \mathrm{~Gy}$ (RBE) vs. $17.0 \pm 16.1 \mathrm{~Gy}(\mathrm{RBE})$, $p<0.001)$.

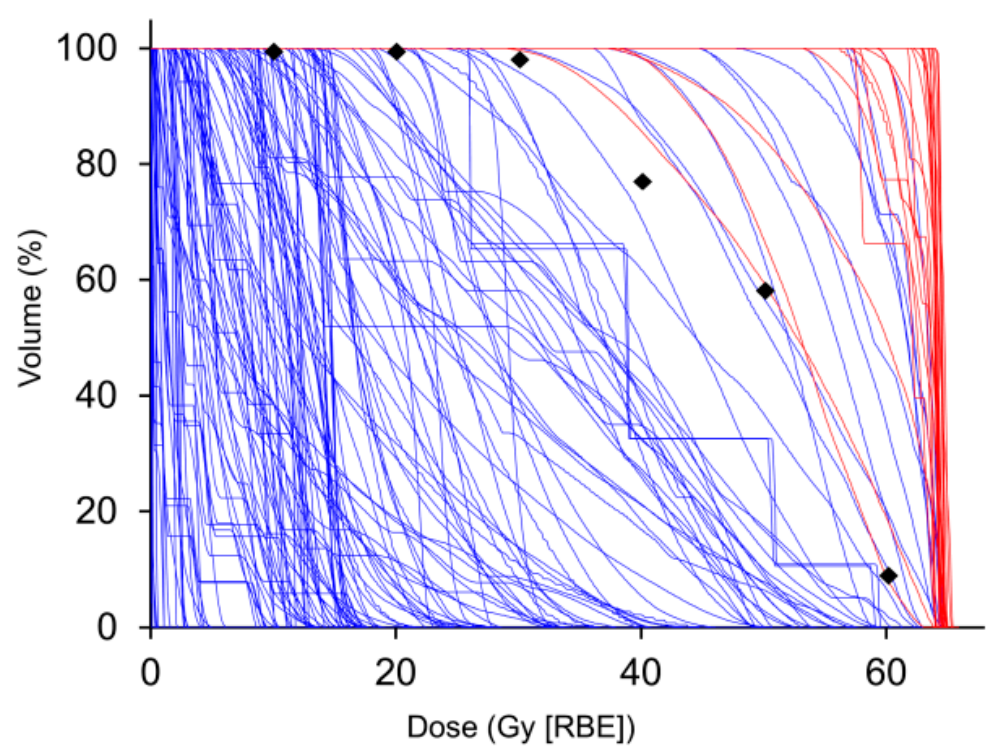

Figure 3. Cumulative dose-volume histograms for the teeth of patients with head and neck nonsquamous cell carcinoma treated with carbon ion radiotherapy. Red and blue lines indicate lost teeth $(n=24)$ and remaining teeth $(n=146)$, respectively. Diamonds show the cut-off values used to distinguish the two groups at every $10 \mathrm{~Gy}$ (relative biological effectiveness), which was calculated using receiver operating characteristic analysis.

Having shown clearly that carbon ion irradiation contributes to tooth loss, we sought to determine the dosimetric parameters that predict tooth loss post-CIRT. To this end, we performed ROC curve analysis using the dose-volume data for the teeth (Table 2). Among the dose-volume parameters analyzed (i.e., V10, V20, V30, V40, V50, and V60), V50 showed the highest accuracy (i.e., 0.95 ; cut-off, $58.1 \%$ ) for predicting tooth loss post-CIRT. The 5 year cumulative incidence of tooth loss using the cutoff values were $0 \%$ versus $30.2 \%$ for $\mathrm{V} 10,0 \%$ versus $49.8 \%$ for $\mathrm{V} 20,0 \%$ versus $63.1 \%$ for $\mathrm{V} 30,0 \%$ versus $63.1 \%$ for $\mathrm{V} 40,0 \%$ versus $66.9 \%$ for V50, $0 \%$ versus $64.9 \%$ for V60, and $0 \%$ versus $63.1 \%$ for V60. More than half of the teeth that exceeded the V30-60 cutoff values fell out within 5 years (Figure 4).

Table 2. Receiver operating characteristic curve analysis to predict tooth loss after carbon ion radiotherapy.

\begin{tabular}{ccccccc}
\hline Parameter & V10 & V20 & V30 & V40 & V50 & V60 \\
\hline Cut-off value & $99.9 \%$ & $99.1 \%$ & $97.8 \%$ & $78.1 \%$ & $58.1 \%$ & $7.67 \%$ \\
Sensitivity & 1.00 & 1.00 & 1.00 & 1.00 & 1.00 & 1.00 \\
Specificity & 0.66 & 0.86 & 0.92 & 0.92 & 0.94 & 0.93 \\
Accuracy & 0.71 & 0.88 & 0.94 & 0.94 & 0.95 & 0.94 \\
\hline
\end{tabular}


(A)

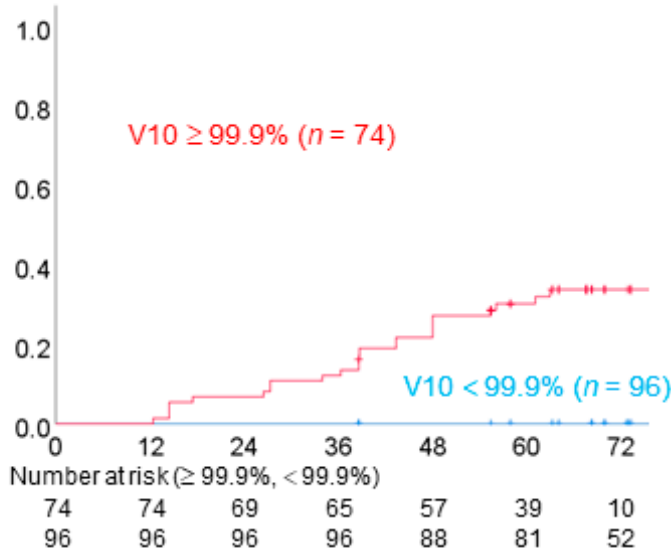

(C)

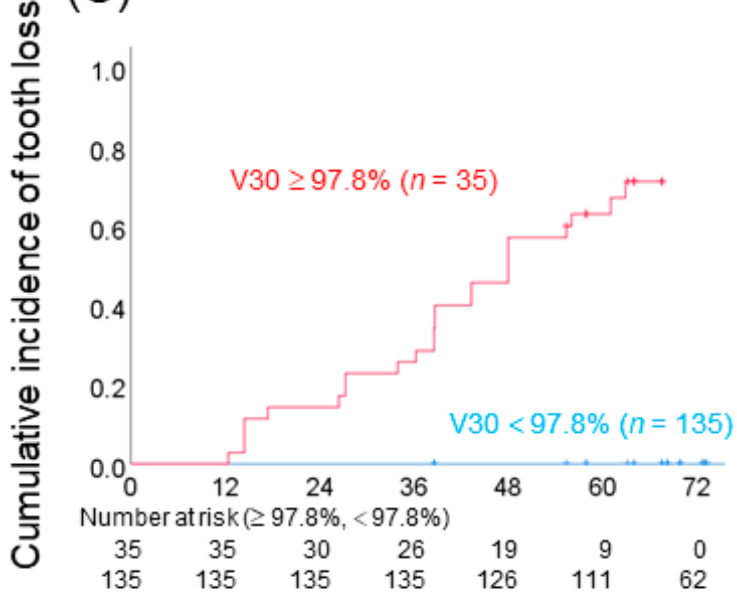

$(\mathrm{E})$

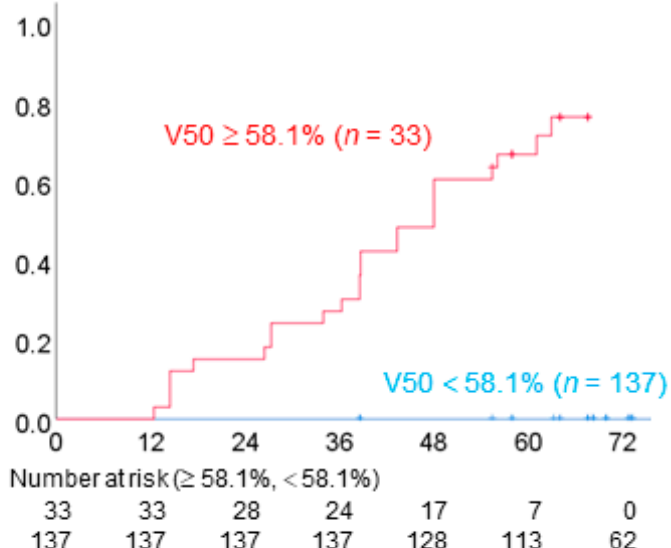

(B)

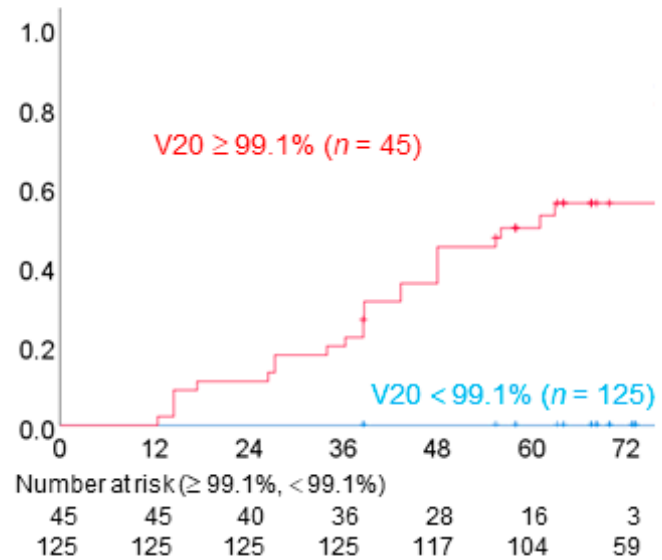

(D)

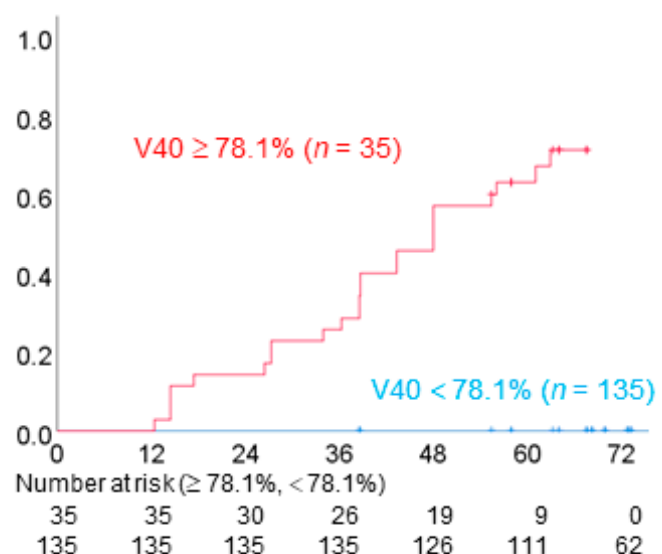

$(\mathrm{F})$

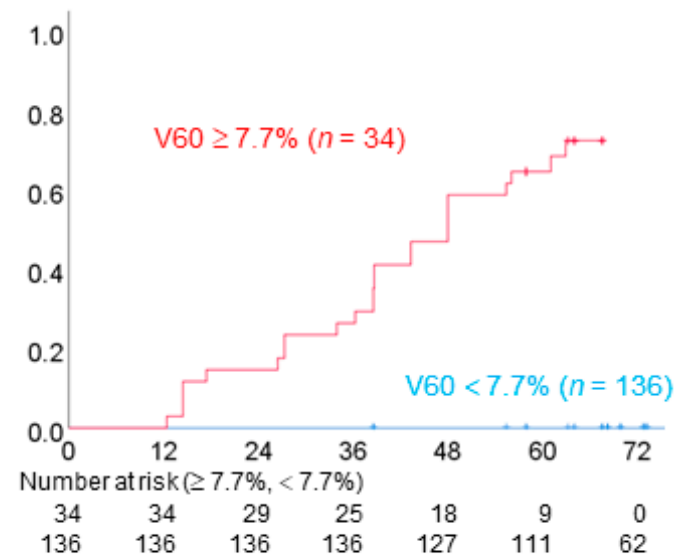

Time after carbon-ion radiotherapy (months)

Figure 4. Cumulative incidence of tooth loss after carbon ion radiotherapy, which was stratified according to cut-off values calculated from receiver operating characteristic analysis. The cutoff values were $99.9 \%, 99.1 \%, 97.8 \%, 78.1 \%, 58.1 \%$, and 7.67\% for V10-60, respectively. (A) V10. (B) V20. (C) V30. (D) V40. (E) V50. (F) V60.

\section{Discussion}

Here, we demonstrate the incidence of tooth loss in a prospective cohort of patients with head and neck tumors treated with CIRT. We also showed that the volume of the irradiated tooth predicts tooth loss post-CIRT throughout the dose range and that V50 is 
the best dose-volume parameter for this purpose. To the best of our knowledge, this study is the first to report the incidence of tooth loss post-CIRT and to identify risk-predictive dosimetric parameters. These data will aid clinical management of patients with head and neck tumors treated with CIRT.

Tooth loss post-radiotherapy has not been fully investigated [22-24]. Indeed, few studies report tooth loss post-radiotherapy. Beesley et al. compared the incidence of tooth loss in patients after intensity-modulated radiotherapy or conventional three-dimensional conformal radiotherapy [25]. In that study, the incidence of tooth loss was comparable between the two radiation modalities. They also found that the incidence of tooth loss was higher in the second year than in first year post-treatment. They concluded that extending the follow-up period to $3-5$ years is required to obtain a complete picture of tooth loss postradiotherapy. From this perspective, our data indicate that tooth loss occurs at a median of approximately 3 years post-CIRT and that most events occur within 5 years (Figure 2). We found that the 5 year cumulative incidence was $13.3 \%$, but the figure of $13.3 \%$ is not relevant because we only enrolled cases in which teeth were irradiated (i.e., 10 patients were excluded because they did not receive irradiation to the tooth). Importantly, the median time of tooth loss was 38.6 months, indicating that long-term follow-up is necessary. We recommend long-term dental visits in cases where teeth have been irradiated. Walker et al. analyzed the association between photon dose and damage to teeth in 93 patients with head and neck tumors; they found that damage to the teeth was significantly higher at a dose of 30 Gy or more [26]. These results are consistent with our own data showing that the accuracy for the ROC curve analysis-based prediction of tooth loss post-CIRT reached approximately $95 \%$ at V30, V40, V50, and V60, whereas the accuracy was below $90 \%$ at V10 and V20 (Table 2). Lieshout et al. reported that the first signs of deterioration of hard teeth tissue are visible within 3 months after radiotherapy in the head and neck region. The most common damage to teeth manifested as widespread areas of porosity in the enamel, crater formation (with exposure of subsurface enamel), loss of large parts of the surface enamel, and loss of full enamel coverage exposing the underlying dentine [27]. Although the mechanism of tooth loss after CIRT is unknown, the first symptoms are often tooth agitation and pain. During photon radiotherapy for head and neck SCC, the salivary glands are irradiated because the lymph node areas are irradiated. As a result, tooth decay due to salivary gland depletion is common $[26,28]$. During CIRT for head and neck NSCC, the lymph node area is usually not irradiated as mentioned in the Materials and Methods Section. There was very little damage to the salivary glands and xerostomia rarely occurred in CIRT. Differences in salivary gland damage are not due to differences in radiation quality between photon radiotherapy and CIRT, but rather due to the differences in the primary tumor site and histology between head and neck NSCC and SCC.

The strength of this study is that we, for the first time, have analyzed the dose-volume relationship in individual teeth. Previous dose-volume studies delineated the whole maxillary bone as the object of analysis and used osteonecrosis or sequestrum as the endpoint. For example, Sasahara et al. reported risk factors for maxillary osteoradionecrosis postCIRT [17]. In that study, they found that V50 was an independent risk factor for maxillary osteonecrosis. Using such dose-volume data to estimate the site and number of teeth lost is difficult. Since CIRT has a steep dose distribution, detailed dose-volume data for each individual tooth will help clinical management prior to initiation of treatment. From a practical perspective, practitioners can use the risk-predictive parameters demonstrated in this study to (i) spare specific teeth that are important for the patient's QOL (i.e., a dose of $50 \mathrm{~Gy}$ (RBE) should not cover more than half of the circumference of the root of the tooth); or (ii), before CIRT is started, to extract the tooth predicted to be lost as this can prevent chronic pain induced by sequestrum. During photon radiotherapy, tooth extraction increases the risk for osteonecrosis. Therefore, tooth extraction prior to radiation therapy is considered [29]. We believe that tooth extraction is also a risk for osteonecrosis after CIRT. From this point of view, it is desirable to extract the necessary teeth before treatment. 
However, it should be noted that extracting the tooth before CIRT can delay the start of treatment.

In addition to radiation, causes of tooth loss include caries and periodontal disease [30-32]. Furthermore, tumor invasion into the maxilla may be a factor that affects tooth loss after treatment. This study did not examine these factors. However, none of the patients lost teeth due to extractions caused by tooth decay in this study. In order to clarify the relationship between the irradiated dose and tooth loss, we excluded patients who did not have irradiated teeth. Of the 25 teeth lost, one was excluded from the analysis because it was clearly an outlier in terms of the irradiated dose; therefore, we assumed that loss of this tooth was caused by factors other than radiation. Regarding loss of the other 24 teeth, we noted that the maxillary bone that supported these teeth disappeared. These changes occurred in line with the distribution of high-dose irradiation in CIRT (Figure 1B) and were uncommon with photon radiotherapy. Clinically, we believe that tooth loss is related to the loss of maxillary bone and evaluated the dose of only the root part and not the crown part in this study. The dosimetric parameters for the root part predicted tooth loss with high accuracy. If factors other than irradiation, such as caries and periodontal disease, had a greater influence, the relationship between dosimetric parameters and tooth loss would not be so clear. Taken together, we consider that factors other than irradiation had a small effect on the loss of the teeth analyzed herein. If these cutoffs can be adhered to, then tooth loss can be prevented in most cases.

The study has several limitations: first, the number of patients was small. Validation with a larger cohort is warranted. Second, confounding factors such as caries, periodontal disease, and tumor invasion were not analyzed. However, it is useful to predict with high accuracy which teeth will disappear after treatment from a clinical perspective. Third, only the maxillary teeth were included and not the mandibular teeth. This is because more than half of tumors are located in the maxilla sinus and nasal cavity and there were few cases in which the mandible was irradiated.

\section{Conclusions}

We report for the first time the incidence of tooth loss in patients with head and neck tumors treated with CIRT and identify risk-predictive dosimetric parameters. The data will be useful for the clinical management of patients with head and neck tumors treated with CIRT.

Supplementary Materials: The following are available online at https:/ /www.mdpi.com/article/10 .3390 /radiation1030017/s1, Figure S1: (A) Cumulative dose-volume histograms for the 25 lost teeth. The red line indicates the tooth excluded from the analysis. (B) Panoramic radiographs taken before carbon ion radiotherapy. The yellow arrow shows the tooth excluded from analysis.

Author Contributions: Conceptualization, N.K., M.S., and Y.K.; methodology, N.K., M.S., and Y.K.; investigation, N.K., H.K., M.A., A.A., H.S., A.M., N.O., and T.K.; writing—original draft preparation, N.K. and T.O. (Takahiro Oike); writing-review and editing, T.O. (Takahiro Oike); supervision, K.S., J.-i.S., S.Y., K.C., and T.O. (Tatsuya Ohno); project administration, N.K.; All authors have read and agreed to the published version of the manuscript.

Funding: This research received no external funding.

Institutional Review Board Statement: The study was conducted according to the guidelines of the Declaration of Helsinki and approved by the Institutional Review Board of Gunma University (ID: HS2019-191).

Informed Consent Statement: Informed consent was obtained from all subjects involved in the study.

Data Availability Statement: The data presented in this study are available upon request from the corresponding author. The data are not publicly available because they contain information that could compromise the privacy of the study participants.

Acknowledgments: The authors thank Masashi Ohishi and Taku Miyabe at the Accelerator Engineering Corporation, Chiba, Japan, for technical support. 
Conflicts of Interest: The authors declare no conflict of interest.

\section{References}

1. Delaney, G.; Jacob, S.; Featherstone, C.; Barton, M. The role of radiotherapy in cancer treatment: Estimating optimal utilization from a review of evidence-based clinical guidelines. Cancer 2005, 104, 1129-1137. [CrossRef] [PubMed]

2. Strojan, P.; Hutcheson, K.A.; Eisbruch, A.; Beitler, J.J.; Langendijk, J.A.; Lee, A.W.M.; Corry, J.; Mendenhall, W.M.; Smee, R.; Rinaldo, A.; et al. Treatment of late sequelae after radiotherapy for head and neck cancer. Cancer Treat. Rev. 2017, 59, 79-92. [CrossRef] [PubMed]

3. Schlaff, C.D.; Krauze, A.; Belard, A.; O'Connell, J.J.; Camphausen, K.A. Bringing the heavy: Carbon ion therapy in the radiobiological and clinical context. Radiat. Oncol. 2014, 9, 88. [CrossRef] [PubMed]

4. Kanai, T.; Furusawa, Y.; Fukutsu, K.; Itsukaichi, H.; Eguchi-Kasai, K.; Ohara, H. Irradiation of Mixed Beam and Design of Spread-Out Bragg Peak for Heavy-Ion Radiotherapy. Radiat. Res. 1997, 147, 78. [CrossRef]

5. Kamada, T.; Tsujii, H.; Blakely, E.A.; Debus, J.; De Neve, W.; Durante, M.; Jäkel, O.; Mayer, R.; Orecchia, R.; Pötter, R.; et al. Carbon ion radiotherapy in Japan: An assessment of 20 years of clinical experience. Lancet Oncol. 2015, 16, e93-e100. [CrossRef]

6. Miyasaka, Y.; Komatsu, S.; Abe, T.; Kubo, N.; Okano, N.; Shibuya, K.; Shirai, K.; Kawamura, H.; Saitoh, J.-I.; Ebara, T.; et al. Comparison of Oncologic Outcomes between Carbon Ion Radiotherapy and Stereotactic Body Radiotherapy for Early-Stage Non-Small Cell Lung Cancer. Cancers 2021, 13, 176. [CrossRef]

7. Imai, R.; Kamada, T.; Araki, N.; Abe, S.; Iwamoto, Y.; Ozaki, T.; Chuman, H.; Hiraga, H.; Hiruma, T.; Kamade, N.; et al. Carbon Ion Radiation Therapy for Unresectable Sacral Chordoma: An Analysis of 188 Cases. Int. J. Radiat. Oncol. 2016, 95, 322-327. [CrossRef]

8. Kawamura, H.; Kubo, N.; Sato, H.; Mizukami, T.; Katoh, H.; Ishikawa, H.; Ohno, T.; Matsui, H.; Ito, K.; Suzuki, K.; et al. Moderately hypofractionated carbon ion radiotherapy for prostate cancer; a prospective observational study "GUNMA0702". BMC Cancer 2020, 20, 75. [CrossRef]

9. Kawashiro, S.; Yamada, S.; Okamoto, M.; Ohno, T.; Nakano, T.; Shinoto, M.; Shioyama, Y.; Nemoto, K.; Isozaki, Y.; Tsuji, H.; et al. Multi-institutional Study of Carbon-ion Radiotherapy for Locally Advanced Pancreatic Cancer: Japan Carbon-ion Radiation Oncology Study Group (J-CROS) Study 1403 Pancreas. Int. J. Radiat. Oncol. 2018, 101, 1212-1221. [CrossRef]

10. Saitoh, J.-I.; Koto, M.; Demizu, Y.; Suefuji, H.; Ohno, T.; Tsuji, H.; Okimoto, T.; Shioyama, Y.; Nemoto, K.; Nakano, T.; et al. A Multicenter Study of Carbon-Ion Radiation Therapy for Head and Neck Adenocarcinoma. Int. J. Radiat. Oncol. 2017, 99, 442-449. [CrossRef]

11. Sulaiman, N.S.; Demizu, Y.; Koto, M.; Saitoh, J.-I.; Suefuji, H.; Tsuji, H.; Ohno, T.; Shioyama, Y.; Okimoto, T.; Daimon, T.; et al. Multicenter Study of Carbon-Ion Radiation Therapy for Adenoid Cystic Carcinoma of the Head and Neck: Subanalysis of the Japan Carbon-Ion Radiation Oncology Study Group (J-CROS) Study (1402 HN). Int. J. Radiat. Oncol. 2018, 100, 639-646. [CrossRef] [PubMed]

12. Suefuji, H.; Koto, M.; Demizu, Y.; Saitoh, J.-I.; Shioyama, Y.; Tsuji, H.; Okimoto, T.; Ohno, T.; Nemoto, K.; Nakano, T.; et al. A Retrospective Multicenter Study of Carbon Ion Radiotherapy for Locally Advanced Olfactory Neuroblastomas. Anticancer Res. 2018, 38, 1665-1670. [CrossRef]

13. Mizoguchi, N.; Tsuji, H.; Toyama, S.; Kamada, T.; Tsujii, H.; Nakayama, Y.; Mizota, A.; Ohnishi, Y. Carbon-ion radiotherapy for locally advanced primary or postoperative recurrent epithelial carcinoma of the lacrimal gland. Radiother. Oncol. 2015, 114, 373-377. [CrossRef] [PubMed]

14. Laura, Q.M.; Chow, M.D. Head and Neck Cancer. N. Engl. J. Med. 2020, 382, 60-72. [CrossRef]

15. Forastiere, A.A.; Koch, W.M.; Trotti, A.; Sidransky, D. Head and Neck Cancer. N. Engl. J. Med. 2001, 345, 1890-1900. [CrossRef]

16. Shirai, K.; Saitoh, J.-I.; Musha, A.; Abe, T.; Kobayashi, D.; Takahashi, T.; Tamaki, T.; Kawamura, H.; Takayasu, Y.; Shino, M.; et al. Prospective observational study of carbon-ion radiotherapy for non-squamous cell carcinoma of the head and neck. Cancer Sci. 2017, 108. [CrossRef]

17. Sasahara, G.; Koto, M.; Ikawa, H.; Hasegawa, A.; Takagi, R.; Okamoto, Y.; Kamada, T. Effects of the dose-volume relationship on and risk factors for maxillary osteoradionecrosis after carbon ion radiotherapy. Radiat. Oncol. 2014, 9, 92. [CrossRef]

18. Hasegawa, A.; Mizoe, J.-E.; Mizota, A.; Tsujii, H. Outcomes of visual acuity in carbon ion radiotherapy: Analysis of dose-volume histograms and prognostic factors. Int. J. Radiat. Oncol. 2006, 64, 396-401. [CrossRef]

19. Kubo, N.; Kubota, Y.; Kawamura, H.; Oike, T.; Sakai, M.; Kumazawa, T.; Miyasaka, Y.; Okazaki, S.; Kobayashi, D.; Sato, H.; et al. Dosimetric parameters predictive of nasolacrimal duct obstruction after carbon-ion radiotherapy for head and neck carcinoma. Radiother. Oncol. 2019, 141, 72-77. [CrossRef]

20. Shirai, K.; Fukata, K.; Adachi, A.; Saitoh, J.-I.; Musha, A.; Abe, T.; Kanai, T.; Kobayashi, D.; Shigeta, Y.; Yokoo, S.; et al. Dosevolume histogram analysis of brainstem necrosis in head and neck tumors treated using carbon-ion radiotherapy. Radiother. Oncol. 2017, 125, 36-40. [CrossRef]

21. Kanai, T.; Endo, M.; Minohara, S.; Miyahara, N.; Koyama-Ito, H.; Tomura, H.; Matsufuji, N.; Futami, Y.; Fukumura, A.; Hiraoka, T.; et al. Biophysical characteristics of HIMAC clinical irradiation system for heavy-ion radiation therapy. Int. J. Radiat. Oncol. 1999, 44, 201-210. [CrossRef]

22. Vissink, A.; Jansma, J.; Spijkervet, F.K.L.; Burlage, F.R.; Coppes, R.P. Oral Sequelae of Head and Neck Radiotherapy. Crit. Rev. Oral Biol. Med. 2003, 14, 199-212. [CrossRef] 
23. Bhandari, S.; Soni, B.W.; Bahl, A.; Ghoshal, S. Radiotherapy-induced oral morbidities in head and neck cancer patients. Spec. Care Dent. 2020, 40, 238-250. [CrossRef] [PubMed]

24. Kielbassa, A.M.; Hinkelbein, W.; Hellwig, E.; Meyer-Lückel, H. Radiation-related damage to dentition. Lancet Oncol. 2006, 7, 326-335. [CrossRef]

25. Beesley, R.; Rieger, J.; Compton, S.; Parliament, M.; Seikaly, H.; Wolfaardt, J. Comparison of tooth loss between intensitymodulated and conventional radiotherapy in head and neck cancer patients. J. Otolaryngol. Head Neck Surg. 2012, 41, 389-395. [CrossRef]

26. Walker, M.P.; Wichman, B.; Cheng, A.-L.; Coster, J.; Williams, K.B. Impact of radiotherapy dose on dentition breakdown in head and neck cancer patients. Pract. Radiat. Oncol. 2011, 1, 142-148. [CrossRef] [PubMed]

27. Lieshout, H.F.J.; Bots, C.P. The effect of radiotherapy on dental hard tissue-A systematic review. Clin. Oral Investig. 2014, 18, 17-24. [CrossRef]

28. Pinna, R.; Campus, G.; Cumbo, E.; Mura, I.; Milia, E. Xerostomia induced by radiotherapy: An overview of the physiopathology, clinical evidence, and management of the oral damage. Ther. Clin. Risk Manag. 2015, 11, 171-188. [CrossRef]

29. Koga, D.H.; Salvajoli, J.V.; Alves, F.A. Dental extractions and radiotherapy in head and neck oncology: Review of the literature. Oral Dis. 2008, 14, 40-44. [CrossRef]

30. Aida, J.; Ando, Y.; Akhter, R.; Aoyama, H.; Masui, M.; Morita, M. Reasons for Permanent Tooth Extractions in Japan. J. Epidemiol. 2006, 16, 214-219. [CrossRef]

31. Hirotomi, T.; Yoshihara, A.; Ogawa, H.; Miyazaki, H. Tooth-related risk factors for tooth loss in community-dwelling elderly people. Community Dent. Oral Epidemiol. 2012, 40, 154-163. [CrossRef] [PubMed]

32. Ravald, N.; Johansson, C.S. Tooth loss in periodontally treated patients. A long-term study of periodontal disease and root caries. J. Clin. Periodontol. 2012, 39, 73-79. [CrossRef] [PubMed] 